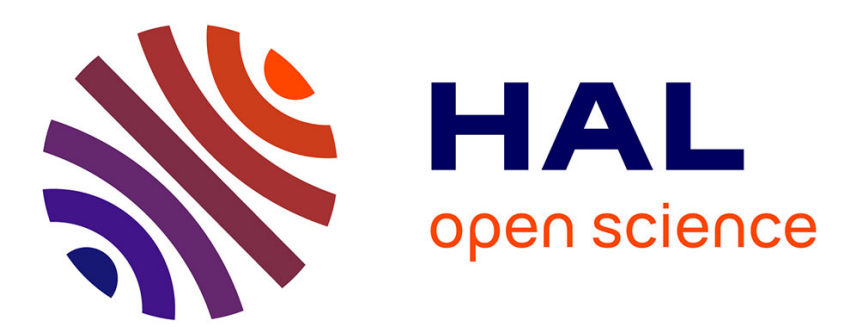

\title{
Automatisation des mesures cinétiques en dynamique dans le cas de la réaction catalytique gaz-solide d'oxydation ménagée du propène
}

\author{
M. Forissier, A. Larchier, L. de Mourgues, M. Perrin, J.-L. Portefaix
}

\section{- To cite this version:}

M. Forissier, A. Larchier, L. de Mourgues, M. Perrin, J.-L. Portefaix. Automatisation des mesures cinétiques en dynamique dans le cas de la réaction catalytique gaz-solide d'oxydation ménagée du propène. Revue de Physique Appliquée, 1976, 11 (5), pp.639-646. 10.1051/rphysap:01976001105063900 . jpa00244099

\section{HAL Id: jpa-00244099 https://hal.science/jpa-00244099}

Submitted on 1 Jan 1976

HAL is a multi-disciplinary open access archive for the deposit and dissemination of scientific research documents, whether they are published or not. The documents may come from teaching and research institutions in France or abroad, or from public or private research centers.
L'archive ouverte pluridisciplinaire HAL, est destinée au dépôt et à la diffusion de documents scientifiques de niveau recherche, publiés ou non, émanant des établissements d'enseignement et de recherche français ou étrangers, des laboratoires publics ou privés. 


\title{
AUTOMATISATION DES MESURES CINÉTIQUES EN DYNAMIQUE DANS LE CAS DE LA RÉACTION CATALYTIQUE GAZ-SOLIDE D'OXYDATION MÉNAGÉE DU PROPẼNE (*)
}

\author{
M. FORISSIER, A. LARCHIER, L. de MOURGUES, M. PERRIN et J.-L. PORTEFAIX
}

Institut de Recherches sur la Catalyse, C. N. R. S., 79, bd du 11 Novembre 1918

69626 Villeurbanne Cédex, France

(Reçu le 9 février 1976, accepté le 4 mai 1976)

\begin{abstract}
Résumé. - Nous avons réalisé un appareil pour l'étude cinétique en dynamique de la réaction catalytique d'oxydation ménagée du propène, pouvant effectuer des séries de mesures sans l'intervention d'un opérateur et suivant un programme préétabli. Le nombre de valeurs expérimentales obtenues s'en trouve considérablement accru, ce qui rend plus sûre l'interprétation des résultats et permet d'envisager un dépouillement statistique. Cette réalisation nous a conduit à concevoir et mettre au point un certain nombre de systèmes originaux que nous décrivons dans ce texte.
\end{abstract}

\begin{abstract}
We have developed an apparatus to study the kinetics of the catalytic reaction of propylene soft oxdation in a flowing system. Following a preestablished program, the apparatus is able to execute a series of measurements without the need for operator interference. In this way we have collected a significantly increased volume of experimental values and we can now envisage a statistical treatment of these values. This development has led us to conceive of and realize several new types of apparatus.
\end{abstract}

1. Introduction. - Les mesures cinétiques constituent un moyen d'investigation essentiel pour les recherches tant fondamentales qu'industrielles sur les réactions chimiques, et tout particulièrement pour celles sur les réactions catalytiques. Elles permettent en effet de déterminer le mécanisme d'une réaction ou sa loi de vitesse, d'évaluer certains paramètres significatifs comme des énergies d'activation, de tester ou de comparer l'efficacité de catalyseurs.

Nous avons réalisé un appareil de mesures cinétiques dans le cas d'une réaction de catalyse hétérogène gaz-solide : l'oxydation ménagée du propène sur des oxydes ou mélanges d'oxydes de métaux de transition. Cette réaction conduit essentiellement à l'acroléine avec comme sous-produits l'acétone, le propanal, 1'éthanal et les produits d'oxydation totale $\left(\mathrm{CO}, \mathrm{CO}_{2}\right.$ et l'eau). Dans les réactions de ce type, l'état du catalyseur évolue avec la composition du mélange réactionnel ; cela nous a conduit à préférer un système dynamique dans lequel un flot continu de réactifs traverse le lit catalytique, à un système statique où, à un instant donné, on met en présence les réactifs et le catalyseur,

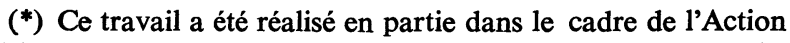
Thématique Programmée du Centre National de la Recherche Scientifique "Intermédiaires à Courte Durée de Vie » (Décision $n^{\circ} 2407$ : Oxydation sélective des Oléfines). puis on suit l'évolution de l'ensemble en fonction du temps ; le système dynamique permet en effet de vérifier qu'un état stationnaire est atteint.

Afin d'améliorer la productivité de l'appareil, nous l'avons conçu de manière à ce qu'il fonctionne de façon automatique d'après un programme préétabli par l'expérimentateur.

2. Principe et critères de validité des mesures cinétiques en dynamique. - Au cours de mesures cinétiques, on détermine des vitesses de réactions dans des conditions opératoires données. Ces vitesses sont calculées à partir des variations de concentration des réactifs et des produits. Un appareil de mesures cinétiques en dynamique comprend donc un circuit réactionnel et un dispositif d'analyse.

Pour que l'on puisse acquérir le maximum d'informations, le circuit réactionnel doit être conçu de façon à ce qu'il soit possible de faire varier les conditions opératoires (débits et concentrations des réactifs, température du réacteur) dans de larges domaines et indépendamment les unes des autres. Il doit aussi permettre de vérifier que la vitesse mesurée est bien celle de la réaction hétérogène et non celle d'une réaction homogène initiée ou non par le catalyseur ; pour cela, Mc Cain et Godin [1] ont montré qu'il suffit de vérifier que la vitesse de la réaction ne change pas avec 
le volume du réacteur. Le circuit réactionnel doit enfin permettre de vérifier que les transferts de matière et de chaleur n'influent pas de façon importante sur la réaction ; d'après Germain [2], il est peu probable que l'on ait des limitations diffusionnelles si on montre que la vitesse de la réaction ne varie pas avec le débit des réactifs et avec le diamètre des grains de catalyseur.

Le dispositif d'analyse quant à lui doit permettre de mesurer la concentration de tous les réactifs et produits afin que l'on puisse faire différents bilans pour s'assurer de la validité des analyses.

3. Description de l'appareil proprement dit. L'appareil que nous avons réalisé en veillant à satisfaire aux exigences énumérées au paragraphe précédent, est schématisé sur la figure 1.

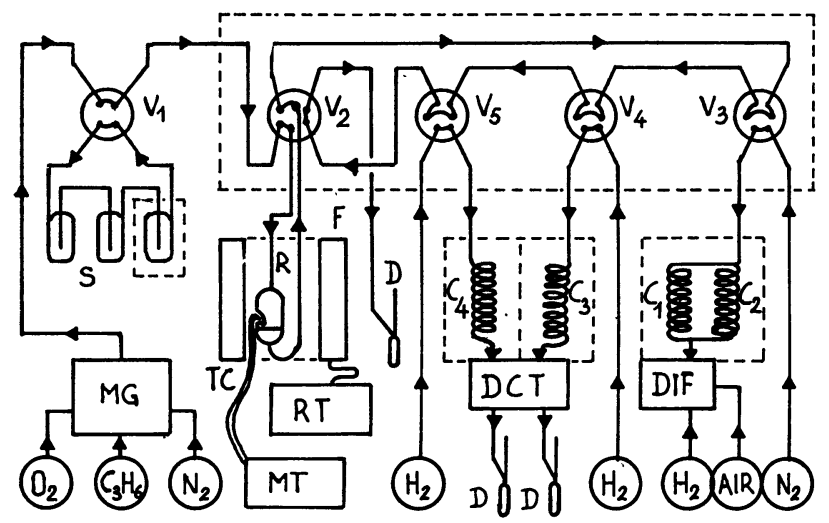

Fig. 1. - L'appareil de mesures cinétiques.
Le circuit réactionnel est constitué successivement d'un mélangeur de gaz (MG) qui par sa conception permet d'imposer un débit, et d'un saturateur (S), placé dans un bain à température constante, qui permettent de réaliser le mélange réactionnel (oxygènepropène-azote, plus éventuellement eau ou acroléine ou tout autre produit dont on veut étudier l'influence ou la dégradation) ainsi que d'un four $(\mathrm{F})$ - dont la température est fixée par un régulateur (RT) et mesurée à l'aide d'un thermocouple (TC) - dans lequel est situé le réacteur $(\mathrm{R})$ contenant le catalyseur. De tous les types de réacteurs dynamiques généralement utilisés pour les mesures cinétiques $[3,4]$, nous avons préféré le microréacteur dynamique différentiel car il permet d'éviter les gradients de température et de concentration dans le lit catalytique ; le réacteur dynamique à circulation qui lui aussi permet d'éviter ces gradients, est d'une technologie plus complexe; de plus, dans ce cas, la présence des produits de la réaction en quantité importante complique la loi de vitesse. Cependant le montage réalisé permet de substituer au microréacteur dynamique différentiel un réacteur intégral ou un réacteur à circulation (pour effectuer des mesures à haut taux de conversion) ou un autre microréacteur de géométrie différente (pour l'étude de l'influence du volume).

Une vanne de commutation (V2) permet d'effectuer l'analyse du mélange avant ou après la réaction. Cette analyse est effectuée par un ensemble de chromatographie en phase vapeur composé de :

trois vannes d'injection thermostatées (V3, V4 et V5),

TABLEAU I

Conditions de l'analyse chromatographique

\begin{tabular}{cc} 
Colonne & \multicolumn{1}{c}{$\mathrm{C1}$} \\
Remplissage & $10 \%$ en poids Carbo- \\
& wax 20 M \\
& sur Chromosorb \\
& WHMDS \\
& $60-80$ mesh
\end{tabular}

Diamètre (pouce)

Longueur (m)

Température $\left({ }^{\circ} \mathrm{C}\right)$

Gaz vecteur

Débit gaz vecteur $(1 / \mathrm{h})$

Détection

Temps de rétention (secondes)

$1 / 4$

3

1,8

C2
$20 \%$ en poids $\mathrm{H}_{3} \mathrm{PO}_{4}$
$15 \%$ en poids DEGS
sur Chromosorb
WHMDS
$60-80$ mesh

$1 / 8$

0,1

60

Azote

3,6

Ionisation de flamme

$\begin{array}{lr}\text { Propène } & : 145 \\ \text { Ethanal } & : 318 \\ \text { Ox. Propylène }: 374 \\ \text { Propanal } & : 471 \\ \text { Acétone } & : 525 \\ \text { Acroléine } & : 618\end{array}$

\begin{tabular}{lr}
\multicolumn{1}{c}{ C3 } & \multicolumn{1}{c}{ C4 } \\
Tamis & Porapak Q \\
moléculaire & $50-80$ mesh \\
$5 \AA$ & \\
45-60 mesh &
\end{tabular}

$1 / 4$

$1 / 4$

3

2

70

Hydrogène

3,6

4,8

Conductivité thermique

Oxygène : 70 Air $: 18$

Azote : $84 \quad \mathrm{CO}_{2} \quad: 57$

$\mathrm{CO}: 145 \mathrm{Eau}: 149$

Propène : 325 
- quatre colonnes $(\mathrm{C} 1, \mathrm{C} 2, \mathrm{C} 3$ et $\mathrm{C} 4)$ décrites par le tableau 1 ,

- un détecteur à ionisation de flamme (DIF),

- un détecteur à conductivité thermique (DCT).

L'enchaînement des opérations est le suivant : après que l'on ait fixé les conditions expérimentales, le mélange réactionnel est analysé puis envoyé en continu et avec un débit constant sur le catalyseur, le mélange sortant du réacteur est analysé périodiquement jusqu'à ce qu'un état stationnaire soit atteint ; on modifie alors les conditions et on recommence le cycle.

4. Automatisation de l'appareil. - Le caractère cyclique de ce processus expérimental prédisposait l'appareil à une automatisation complète que nous avons réalisée ; l'organigramme adopté apparaît sur la figure 2 .

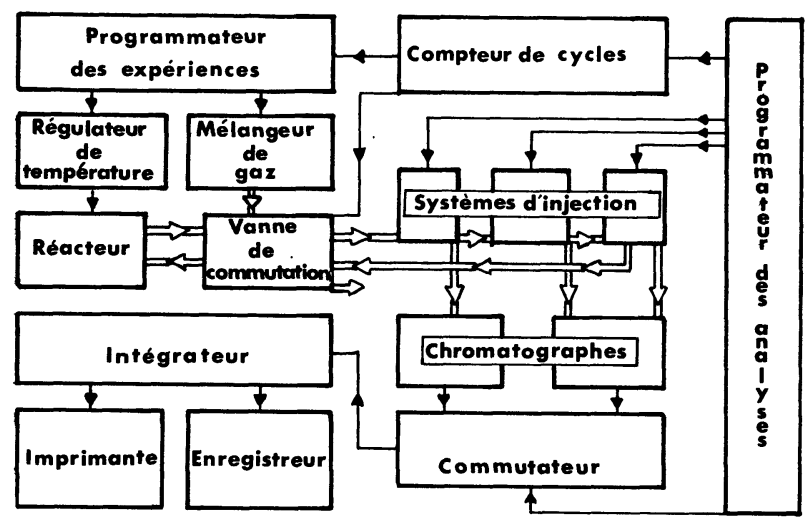

FIG. 2. - Organigramme de l'automatisation.

Le programmateur des analyses effectue des cycles successifs au cours desquels il déclanche, à des instants présélectionnés, les trois vannes d'injection, chacune une fois, et la commutation sur l'intégrateur du détecteur chromatographique en fonction; il incrémente en outre d'une unité le compteur de cycles. Ce compteur, lorsqu'il atteint une valeur $\mathbf{n}$ (choisie par l'opérateur), fait tourner la vanne de commutation, et lorsqu'il atteint une valeur $m$ (supérieure à $n$ et également choisie par l'opérateur) fait revenir la vanne de commutation dans sa position initiale, se remet à zéro et envoie un signal au programmateur des expériences, qui impose alors de nouvelles conditions opératoires au réacteur par l'intermédiaire du régulateur de température et du mélangeur de gaz.

L'intégrateur utilisé est un Vidar Autolab System IV ; les chromatographes sont des Intersmat IGCe $12 \mathrm{M}$ et IGC $12 \mathrm{~F}$.

Le programmateur des expériences est un programmateur à cartes perforées de 24 pistes et avance pas à pas CORECI type $912 \mathrm{CF}$. Une ligne de la carte (1 pas), correspond à un ensemble de conditions opératoires. En effet, comme on le verra plus loin, 18 pistes commandent le mélangeur de gaz et 5 pistes le programmateur de température ; le $24^{\mathrm{e}}$ permet l'avance de la carte.
Parce qu'ils n'existent pas dans le commerce, ou parce que ceux qui existent sont mal adaptés à notre problème, nous avons conçu et mis au point les autres éléments de l'appareil, c'est-à-dire :

- le programmateur des analyses,

- le commutateur,

- le compteur de cycles,

- les systèmes d'injection,

- la vanne de commutation,

- le régulateur de température,

- le mélangeur de gaz.

Le programmateur des analyses, le commutateur et le compteur de cycles sont présentés séparément dans cet exposé par soucis de clarté ; ils constituent en fait un ensemble électronique dont le schéma est donné par la figure 3.

5. Le programmateur des analyses. - Il se compose d'une horloge et de cinq sélecteurs de temps.

L'horloge est synchronisée sur le secteur et, par divisions successives de la fréquence 100 hertz, compte les secondes de 0 à 9999 ; elle commande en outre, par boucle de comptage, des retards temporisés fixes dont nous verrons plus loin l'emploi. Sa remise à zéro peut être obtenue soit manuellement à tout instant, soit automatiquement en fin de cycle.

Les cinq sélecteurs de temps sont à quatre chiffres. Ils permettent de présélectionner les instants notés T0 à $\mathrm{T} 4$, où sont déclanchés, au cours d'un cycle, différentes fonctions.

T0 provoque l'impression des résultats en mémoire dans l'intégrateur (ceux correspondant à la dernière injection du cycle précédent), la commutation du détecteur à conductivité thermique dans la position correspondant à l'analyse sur la colonne $\mathrm{C} 3$ et, après un retard de 16 secondes (temps nécessaire pour que l'intégrateur soit disponible pour l'analyse suivante) l'injection sur cette colonne.

T1 commande le branchement du thermocouple sur l'intégrateur. L'intégration du signal correspondant pendant une durée fixe (T2-T1) permet la mesure de la température du réacteur.

T2 déclenche l'inversion de polarité du détecteur à conductivité thermique pour l'analyse sur la colonne $\mathrm{C} 4$.

T3 provoque l'impression des résultats de l'analyse sur la colonne C3 et de la mesure de température, puis 16 secondes après, l'injection sur la colonne $\mathrm{C} 4$.

T4 déclenche l'impression des résultats de l'analyse précédente, la commutation du détecteur à ionisation de flamme sur l'intégrateur et l'injection, 16 secondes plus tard, sur les colonnes $\mathrm{C} 1$ et $\mathrm{C} 2$.

Les commandes des relais de commutation sont assurées par un étage Darlington. Les circuits extérieurs (vannes d'injection, programmateur des expériences, ...) sont commandés par couplage optoélectrique pour éliminer les parasites et éviter les risques de destruction accidentelle en isolant les circuits. 


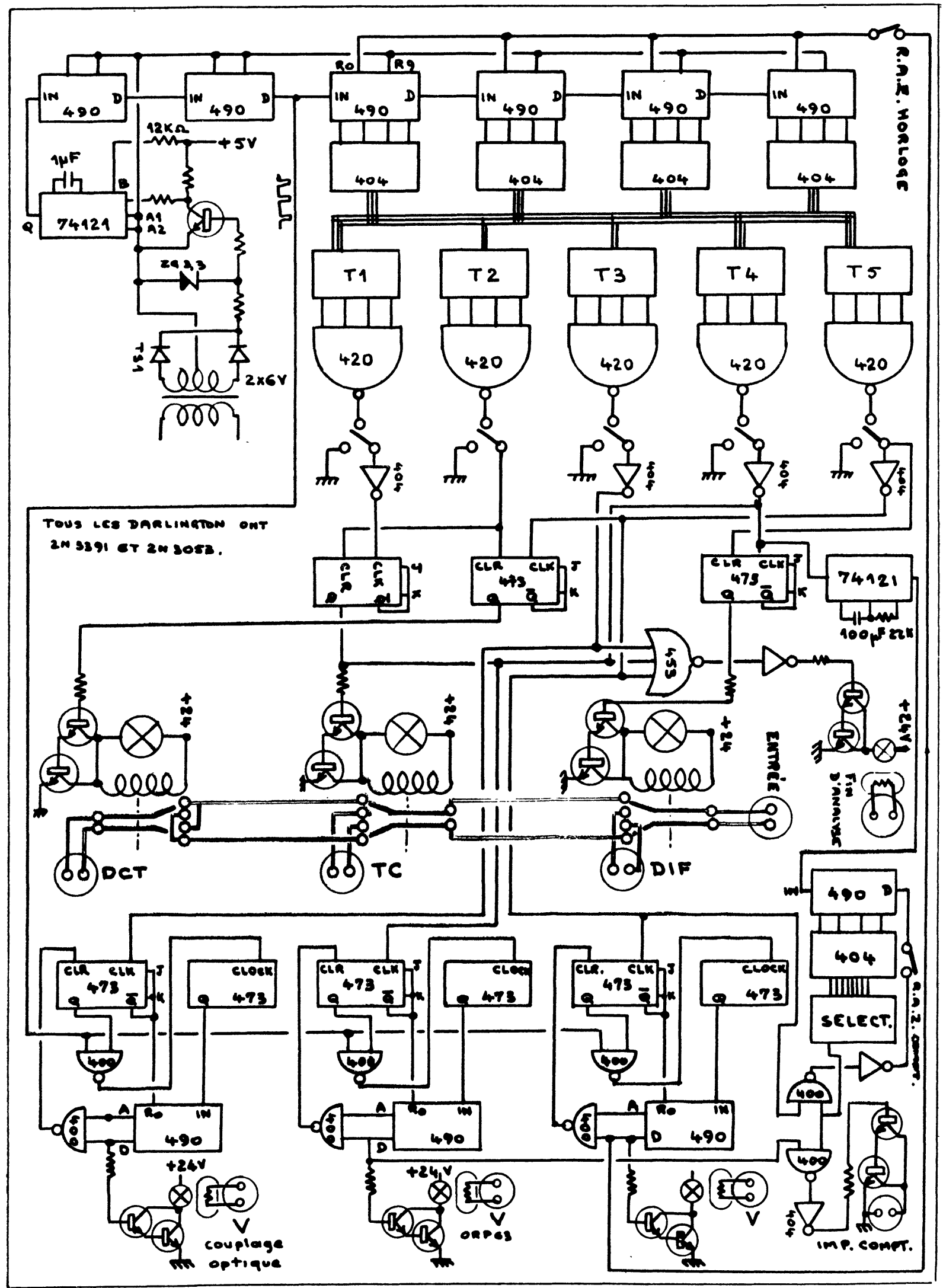

Fig. 3. - Schéma électronique de l'ensemble Programmateur des analyses-Commutateur-Compteur de Cycles. 
Au cours du cycle, des voyants lumineux permettent de connaitre la fonction en cours. Pour des essais non cycliques (étalonnages, mises au point, ...) l'horloge peut être déconnectée, et les différentes fonctions sont alors déclenchées manuellement.

6. Le commutateur de cycles. - Il est destiné à commuter les différentes sources de signaux sur l'intégrateur et à permettre l'inversion de polarité du détecteur à conductivité thermique. Il est constitué de trois relais inverseurs doubles à faible couple de contact (Fig. 3). L'alimentation des enroulements de commande est assurée par le programmateur des analyses.

7. Le compteur de cycles. - Il est constitué d'un compteur et de deux présélecteurs à un chiffre. Au temps T4 de chaque cycle, le compteur reçoit du programmateur des analyses une impulsion qui l'incrémente d'une unité ; lorsqu'il atteint une valeur égale à une de celles affichées sur les présélecteurs, il provoque, par fermeture d'un circuit extérieur, selon le cas, la rotation de la vanne de commutation ou l'avance d'un pas du programmateur des expériences puis dans ce dernier cas, se remet à zéro.

8. Les systèmes d'injection. - L'injection d'un échantillon gazeux dans un chromatographe en phase vapeur se fait le plus souvent à l'aide d'une vanne à six voies et à deux positions $\alpha$ et $\beta$ (Fig. 4). Dans la position $\alpha$, le gaz à analyser balaye une boucle de volume connu, dite boucle d'injection, qui est insérée dans le circuit du gaz vecteur chromatographique (en amont de la colonne) en position $\beta$. En passant d'une position à l'autre de la vanne, il y a interruption des deux circuits gazeux ; une bonne injection exige donc une rotation brusque pour perturber le moins possible les débits chromatographiques.

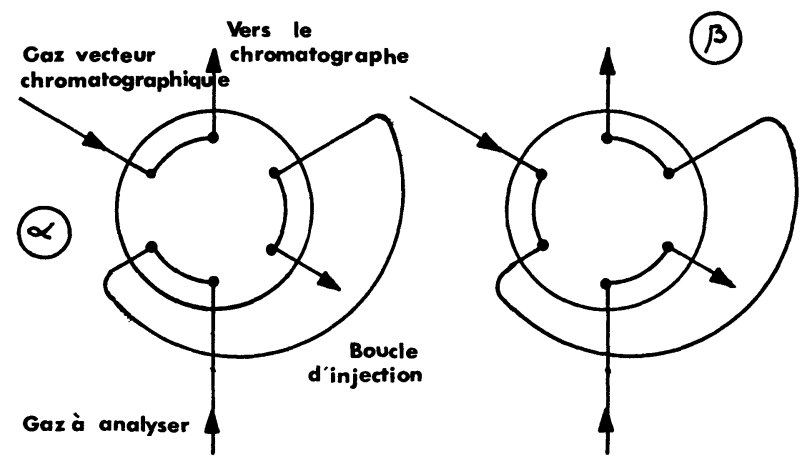

FIG. 4. - Principe des vannes d'injection chromatographiques.

Dans le cas classique d'une vanne à six voies symétrique, le passage de la position $\alpha$ à la position $\beta$ se fait par une rotation de $\pi / 3$; le retour de la position $\beta$ à la position $\alpha$ peut se faire soit par une rotation de $\pi / 3$ en sens inverse, soit par une rotation de $\pi / 3$ dans le même sens. La première solution est la plus couramment employée, mais nous avons préféré utiliser la seconde, car elle se prête plus facilement à l'automatisation.
La vanne à six voies que nous avons fabriquée et utilisée est semblable à celles que l'on trouve dans le commerce. Le dispositif que nous avons réalisé pour la faire tourner brusquement d'un sixième de tour, est représenté sur la figure 5. La vanne d'injection (1) est solidaire de l'axe de la bielle (4). Un moto-réducteur (2) entraîne la rotation de la bielle (5). La pièce (6) articulée sur (5) et s'appuyant sur l'entretoise fixe (7), empêche la rotation de (4). Le ressort (3) se tend jusqu'à ce que (6) échappe à (7). A ce moment (6), libérée, entraîne la vanne sous l'impulsion du ressort (3). Cette rotation s'arrête au bout d'un sixième de tour, lorsque (6) rencontre l'entretoise (7) suivante. La durée de l'injection (position $\beta$ ) doit être suffisante pour que la boucle soit entièrement balayée par le gaz vecteur, et constante. Dans notre réalisation, elle est fixée par la durée d'un sixième de tour du moto-réducteur, soit 10 secondes.

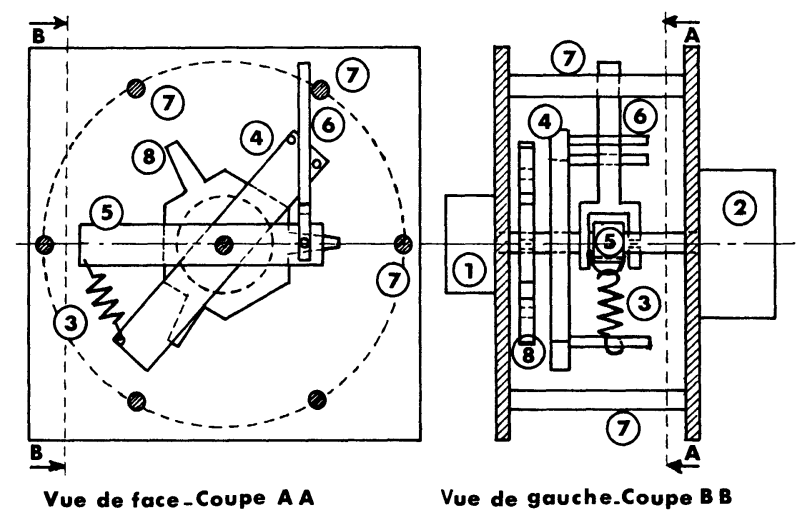

Fig. 5. - Réalisation des systèmes d'injection.

Après le retour en position $\alpha$ qui s'effectue de la même manière, il faut que le moteur s'arrête jusqu'au moment choisi pour l'injection suivante. Son circuit de commande a été conçu en fonction de cette nécessité ; il est représenté sur la figure 6. L'alimentation du moteur dépend de la position de la vanne d'injection par l'intermédiaire de la came (8) de la figure 5 qui lui est solidaire ; cette came agit sur l'inverseur (I). Ainsi en position $\beta$, le moteur est toujours alimenté

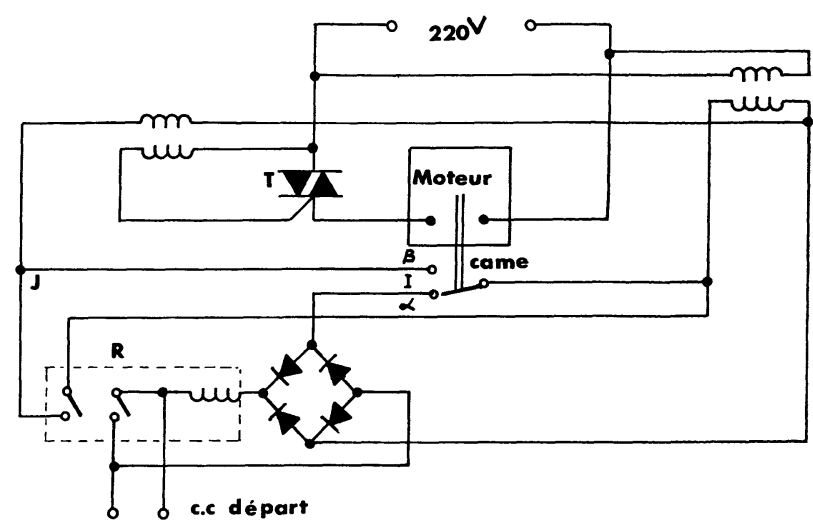

Fig. 6. - Circuit de commande des moteurs des systèmes d'injection. 
(par l'intermédiaire de la branche IJ du circuit); en position $\alpha$, il n'est alimenté qu'après l'impulsion de départ venant du programmateur des analyses et transmise par le relais autocollant (R). Le triac $(T)$ placé dans le circuit électrique du moteur et commandé au passage à zéro de la tension d'alimentation grâce à un signal sinusoïdal synchrone, permet d'éviter les parasites qui apparaissent généralement lors de la rupture ou de l'établissement d'un courant.

9. La vanne de commutation. - Nous avons utilisé pour la vanne de commutation (notée V2) une vanne à six voies identique à celles décrites au paragraphe précédent, et dans laquelle nous avons remplacé la boucle d'injection, par le réacteur (Fig. 1). Son automatisation a été réalisée à l'aide du même système électromécanique, modifié afin que le temps pendant lequel la vanne est en position $\beta$ ne soit pas forcément égal à la durée d'un sixième de tour du moto-réducteur, mais puisse être égal à la durée d'un nombre entier de cycles d'analyses, choisi par l'opérateur. Pour cela nous avons remplacé la came (notée (8) sur la figure 5) agissant sur l'interrupteur (I) tous les tiers de tour par une came analogue mais agissant tous les sixièmes de tours. L'alimentation du moteur est ainsi coupée aussi bien en position $\beta$ qu'en position $\alpha$ et n'est rétablie par le relais autocollant (R) dans les deux cas que sous l'effet de l'impulsion extérieure.

10. Régulateur de température. - Cet appareil est un régulateur proportionnel à sonde de platine, fonctionnant par déphasage d'un pont de Wheatstone en alternatif, et permettant de réguler toute température dans le domaine $0^{\circ} \mathrm{C}-850^{\circ} \mathrm{C}$. Il est relié à cinq pistes du programmateur des expériences, qui correspondent à cinq incréments additifs $\left(5^{\circ} \mathrm{C}, 10^{\circ} \mathrm{C}, 20^{\circ} \mathrm{C}, 40^{\circ} \mathrm{C}\right.$, $80^{\circ} \mathrm{C}$ ) que l'on peut ajouter à la température affichée sur le régulateur. L'ensemble permet donc de programmer successivement un maximum de 32 températures différentes. Ces incréments sont obtenus en introduisant des résistances appropriées dans une branche du pont de Wheatstone de la régulation.

11. Mélangeur de gaz. - Le mélange réactionnel est constitué de 3 gaz. Les 18 commandes du programmateur reliées au mélangeur, correspondent à 18 électrovannes ( 6 pour chaque gaz) qui peuvent mettre en circuit des pertes de charge calibrées en tube capillaire métallique (Fig. 7). Pour un gaz donné, les 6 pertes de charge sont dans des rapports $1,2,4,8,16,32$ et permettent donc de réaliser 63 débits différents (mais identiques pour les $3 \mathrm{gaz}$ ), dans le domaine 2-196 $\mathrm{ml} / \mathrm{mn}$. Ces débits sont parfaitement stables et reproductibles, car ils sont obtenus en appliquant une pression fixe et suffisamment élevée ( 2 à 3 bars) en amont des pertes de charge. L'ensemble permet donc de réaliser $63 \times 63 \times 63$ soit 250047 mélanges gazeux différents, parmi lesquels on peut aisément choisir ceux qui conviennent pour les mesures que l'on veut

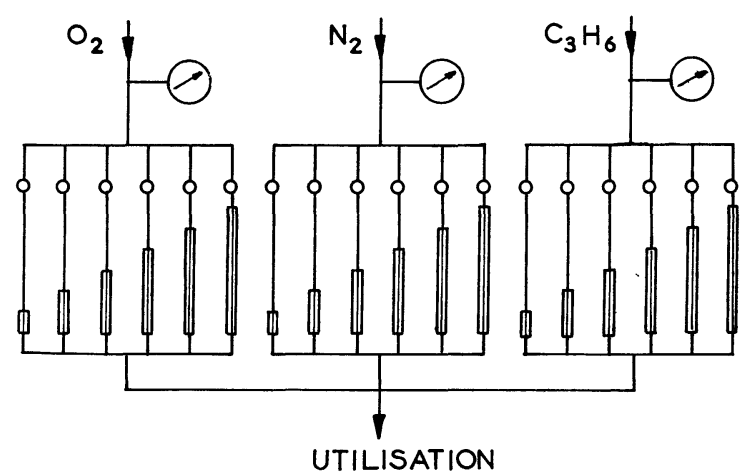

Fig. 7. - Principe du mélangeur de gaz.

réaliser. Ainsi pour mener à bien une étude de l'influence du temps de contact avec un mélange ayant la composition $(10 \%, 20 \%, 70 \%)$, on dispose de 9 valeurs possibles du débit total : $20,40,60,80,100$, 120,140 , et $180 \mathrm{ml} / \mathrm{mn}$. De même pour mesurer l'ordre par rapport au propène dans le cas d'un débit total de $120 \mathrm{ml} / \mathrm{mn}$ et en présence de $10 \%$ d'oxygène, on dispose de 54 possibilités de débits pour le propène $(2,4$, $6, \ldots, 106$ et $108 \mathrm{ml} / \mathrm{mn})$.

Comme on l'a vu au paragraphe 3 , un saturateur placé en aval des pertes de charge permet d'introduire dans le mélange un des produits formés dont on veut étudier l'évolution dans les conditions de la réaction ou l'influence sur cette réaction. Une modification minime du montage permet de faire varier automatiquement cette pression partielle ; il suffit en effet pour cela de ne relier au saturateur que certaines pertes de charge, les débits gazeux issus des autres ne rejoignant le circuit principal qu'après.

12. Fiabilité de l'appareillage et précision des mesures. - Les différents systèmes que nous venons de décrire fonctionnent depuis deux ans sans défaillances graves; ils sont donc d'une bonne fiabilité.

Le tableau II donne les valeurs de l'écart-type sur les surfaces des pics chromatographiques au cours de mesures d'étalonnage ainsi qu'au cours de mesures de la vitesse de la réaction à $430^{\circ} \mathrm{C}$, sur $0,8 \mathrm{~g}$ d'un catalyseur constitué de $0,33 \%$ en poids de molybdène sur de l'alumine, avec un débit de 3,87 1/h d'un mélange réactionnel composé de $23 \%$ d'oxygène, $16,5 \%$ de propène et $60,5 \%$ d'azote. Ces valeurs sont faibles et correspondent à une bonne précision qu'il est difficile de dépasser dans ce type de mesures.

13. Exemples de résultats. - Nous utilisons cet appareil pour déterminer les caractéristiques cinétiques d'oxydes de métaux de transition (catalyseurs d'oxydation ménagée) que nous tentons de relier à d'autres propriétés de ces solides, en particulier leurs propriétés structurales (symétrie du cation, covalence de la liaison métal-oxygène) déterminées à partir de mesures spectroscopiques. 
TABLEAU II

Ecarts-types sur les surfaces des pics chromatographiques

\begin{tabular}{|c|c|c|c|c|c|c|}
\hline Réactif ou produit & $\begin{array}{c}\text { Nombre de } \\
\text { mesures } \\
-\end{array}$ & $\begin{array}{l}\text { Etalonnage } \\
\text { Moyenne des } \\
\text { surfaces } \\
\text { des pics } \\
-\end{array}$ & $\begin{array}{c}\text { Ecart-type } \\
-\end{array}$ & $\begin{array}{c}\text { Nombre de } \\
\text { mesures } \\
-\end{array}$ & $\begin{array}{c}\text { Réaction }\left(^{*}\right) \\
\text { Moyenne des } \\
\text { surfaces } \\
\text { des pics } \\
-\end{array}$ & Ecart-type \\
\hline Oxygène & 9 & 180976 & 197 & 12 & 160046 & 976 \\
\hline Azote & 9 & 729887 & 587 & 12 & 452365 & 2895 \\
\hline $\mathrm{CO}$ & 18 & 92345 & 1053 & 12 & 2958 & 59 \\
\hline $\mathrm{CO}_{2}$ & 19 & 540484 & 1018 & 12 & 2671 & 17 \\
\hline Eau & 23 & 8066 & 137 & 12 & 4092 & 69 \\
\hline Propène & 6 & 623667 & 1375 & 12 & 109671 & 954 \\
\hline Ac. acrylique & 19 & 96908 & 3146 & - & 一 & - \\
\hline Ethanal & 12 & 85859 & 1822 & 12 & 333 & 10 \\
\hline Ox. de Propylène & 13 & 787793 & 10473 & - & - & - \\
\hline Propanal & 7 & 341443 & 1446 & 12 & 208 & 6 \\
\hline Acétone & 14 & 311275 & 5541 & 12 & 81 & 3 \\
\hline Acroléine & 36 & 340349 & 6100 & 12 & 968 & 27 \\
\hline
\end{tabular}

$\left(^{*}\right)$ Oxydation du Propène avec un débit de $64,5 \mathrm{~cm} 3 / \mathrm{mn}$ d'un mélange « 175 torr $\mathrm{O}_{2}-125$ torr $\mathrm{C}_{3} \mathrm{H}_{6}-460$ torr $\mathrm{N}_{2}$ », à $430{ }^{\circ} \mathrm{C}$, sur $0,8 \mathrm{~g}$ d'un catalyseur composé de $0,33 \%$ en poids de molybdène déposé sur de l'alumine (surface $: 23,5 \mathrm{~m}^{2} / \mathrm{g}$ ).

Ainsi, dans le cadre d'une étude de l'influence de l'environnement de l'ion vanadium sur ses propriétés catalytiques pour l'oxydation du propène [5], nous avons comparé des solides constitués de vanadium déposé en quantités variables sur des supports tels que l'alumine, les silices cabosil, $\mathrm{F}$ et Davison, la magnésie ou le rutile. Pour cela, nous avons pour chacun d'entre eux étudié l'influence de la température de la réaction (les autres paramètres ne variant pas) sur les pressions partielles du propène converti et des produits, grandeurs qui dans nos conditions sont proportionnelles à leurs vitesses respectivement de disparition ou de formation. La figure 8 donne à titre d'exemple les résultats obtenus avec $500 \mathrm{mg}$ de l'échantillon VS10, constitué de $1,86 \%$ en poids de vanadium déposé sur de la silice cabosil (surface $=185 \mathrm{~m}^{2} / \mathrm{g}$ ), testé dans le domaine $255-320^{\circ} \mathrm{C}$, avec un débit de $64,5 \mathrm{~cm}^{3} / \mathrm{mn}$ d'un mélange 100 torr d'oxygène-100 torr de propène560 torr d'azote. La programmation des expériences en fonction de la température est faite dans un ordre quelconque pour mettre en évidence un éventuel effet du vieillissement du catalyseur. Dans l'exemple donné, ce mode opératoire a permis de montrer que cet effet ne se produit pas. Le domaine de température exploré aurait pu être beaucoup plus large s'il n'était apparu aux températures les plus élevées, des limitations dues à des phénomènes de transfert de masse ou d'inhibition par les produits de la réaction et mises en évidence par la forme de la courbe qui n'est plus linéaire dans le domaine considéré. Par contre, la pente de la droite obtenue pour les faibles conversions (températures

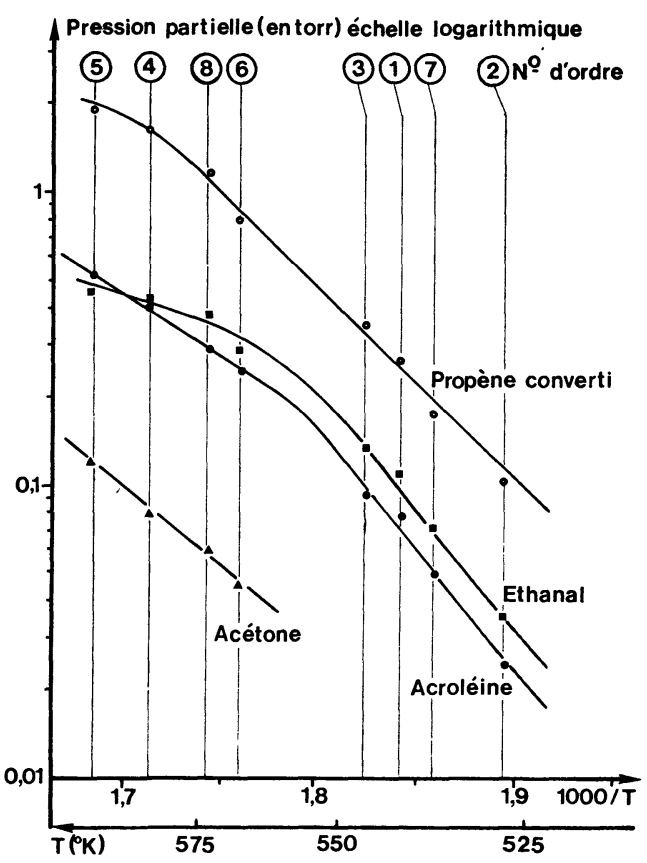

Fig. 8. - Exemple de résultats obtenus au cours d'une étude de l'influence de la température sur les pressions partielles du propène converti et des produits (cas du catalyseur VS10).

les plus basses) est une mesure de l'énergie d'activation apparente de la réaction.

De même, dans le cadre d'une étude de l'influence de l'environnement de l'ion molybdène sur sa sélectivité dans l'oxydation du propène [6], nous avons effectué des mesures analogues sur une série de catalyseurs 
constitués de molybdène déposé en quantités variables sur différents supports. Afin de mieux préciser le mécanisme de l'action catalytique, nous avons pu de plus dans ce cas étudier l'évolution des produits dans les conditions de la réaction, ainsi que leur influence sur la réaction elle-même.

Actuellement, nous effectuons, dans le cas des deux séries de catalyseurs utilisés dans les études citées ci-dessus, des mesures de l'influence des pressions partielles de réactifs (propène et oxygène) sur la vitesse de la réaction. De telles mesures conduisent à la loi de vitesse et permettent de préciser le mécanisme de la réaction. Pour cela nous avons choisi un plan d'expériences (décrit par le tableau III), au cours duquel les pressions partielles de propène et d'oxygène varient indépendamment dans le domaine 25-175 torrs, le débit total étant maintenu à $60 \mathrm{ml} / \mathrm{mn}$. La figure 9 donne les résultats obtenus dans le cas du catalyseur MS3 $(0,21 \%$ en poids de molybdène déposé sur une silice cabosil de $180 \mathrm{~m}^{2} / \mathrm{g}$ ).

\section{TABLEAU III}

Plan d'expériences pour l'étude de l'influence des pressions partielles d'oxygène $\left(\mathrm{Po}_{2}\right)$ et de propène $\left(\mathrm{P}_{\mathrm{C}_{3} \mathrm{H}_{6}}\right)$ sur la vitesse de la réaction d'oxydation du propène (les nombres correspondent aux numéros d'ordre des étapes dans le plan

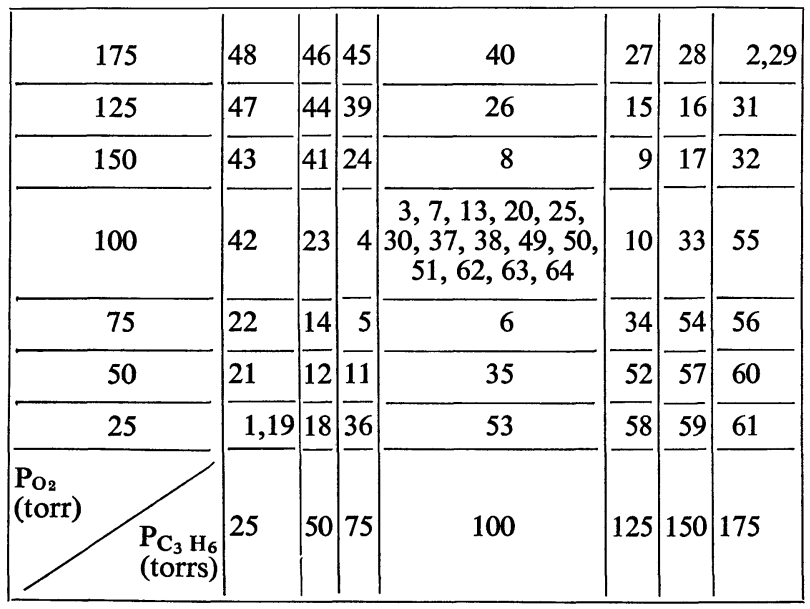

14. Conclusion. - A l'aide de cet appareil nous effectuons toutes les mesures cinétiques souhaitées (essentiellement des déterminations d'ordre de la réac-

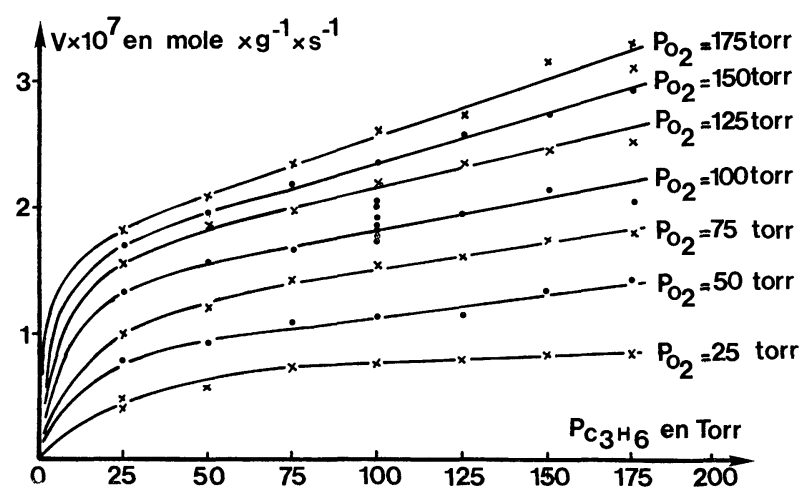

Fig. 9. - Exemple de résultats obtenus au cours d'une étude de l'influence des pressions partielles des réactifs sur la vitesse de la réaction d'oxydation du propène (cas du catalyseur MS3).

tion par rapport à un réactif, d'énergies d'activation, de mécanismes réactionnels, des études de l'influence des produits, de leur dégradation), après que l'on se soit assuré de leur validité par quelques vérifications simples.

L'automatisation permet de le faire fonctionner sans interruption sauf pour charger ou décharger le réacteur ; on évite ainsi d'entacher les mesures d'erreur par une possible évolution du catalyseur due à une interruption des mesures au milieu d'une série ; cela permet de plus de faire des études de vieillissement pendant des durées plus longues ; cela augmente surtout considérablement le nombre des mesures obtenues pendant une période donnée, d'où une plus grande sécurité dans l'interprétation des résultats.

L'automatisation que nous avons réalisée est une étape vers un ensemble plus élaboré couplé à un ordinateur ; ce couplage est techniquement facile à réaliser à partir de notre appareil. L'ordinateur devrait permettre de traiter directement les données expérimentales, c'est-à-dire de calculer les vitesses de réaction à partir des surfaces des pics chromatographiques. Par la suite il pourrait faciliter l'interprétation même des résultats en recherchant parmi une série de mécanismes cinétiques (représentés par des lois mathématiques) proposés par l'opérateur celui qui est le plus probable, voire même indiquer un plan d'expériences à faire au vu des premiers résultats, afin de mieux exécuter cette recherche.

\section{Bibliographie}

[1] Mc Cain, C. C. et Godin, G. W., Nature 202 (1964) 692.

[2] Germain, J. E., La Catalyse au Laboratoire et dans l'Industrie (Masson et Cie, Paris), 1967, p. 45.

[3] Kiperman, S. L., Kinetika i Kataliz 13 (1972) 625.

[4] Doraiswamy, L. K. et Tajbl, D. G., Catalysis Reviews 10 (1975) 77.
[5] Figueras, F., Forissier, M., Portefaix, J. L. e t Rosa Brusin, M., $2^{\text {e }}$ séminaire Franco-Soviétique sur la Catalyse, Kiev, septembre 1974.

[6] Che, M., Figueras, F., Forissier, M., MaC Ateer, J., Perrin, M., Portefaix, J. L. et Praliaud, H., $6^{\mathrm{e}}$ Congrès International de Catalyse, Londres, juillet 1976. 